\title{
Geocomputation with R
}

Lovelace, R.; Nowosad, J. \& Muenchow, J., Geocomputation with R., CRC Press, 2019. ISBN-10: 1138304514, ISBN-13: 978-1138304512

\section{Javier Buenaño Guerra Consultor Data Science (Ecuador)}

El libro titulado Geocomputation with $R$, se divide en tres partes: a) Fundamentos vinculados con los datos geográficos, b) Extensiones, que cubren técnicas avanzadas; $\mathrm{y}, \mathrm{c})$ Aplicaciones a problemas del mundo real.

El desarrollo de contenidos del libro es progresivo, permitiendo al lector adentrarse en el mundo de la geocomputación. En el primer capítulo se aborda la estructura de los objetos espaciales manejados en $\mathrm{R}$, tanto para datos con modelo vectorial como ráster. Los denominados "simple features" consituye un modelo de datos jerárquicos que representan un amplio espectro de tipos de geometría y que son usados a lo largo del libro, para ejecutar una cantidad importante de operaciones espaciales. El modelo mencionado tiene la ventaja de que puede ser usado con otras librerías de $\mathrm{R}$, sin necesidad de realizar una transformación previa; y, además facilita la importación y exportación.

Con respecto a los datos con modelo ráster, el libro plantea el uso de la ya conocida librería raster, cuyas funciones han sido ampliamente tratadas en otras publicaciones.

La primera parte del libro, tiene una pequeña sección en donde se hace referencia a la utilización de los diferentes sistemas de referencia espacial en R. A continuación, incluye un resumen de las principales acciones que se pueden ejecutar a nivel de atributos. Las funciones de la librería dplyr pueden ser usadas sin ninguna dificultad.

Se abordan las principales operaciones espaciales de datos vectoriales como: relaciones topológicas, join espacial, sobreposición, agregación espacial, distancias, Para el caso de datos ráster, se incluyen operaciones como: selección espacial y álgebra de mapas (operaciones: locales, focales, zonales y globales).

Se incluyen operaciones geométricas en datos vectoriales, como simplificación de geometría, obtención de centróides, buffers, transformación afín, recorte, agregación espacial y transformación entre geometrías. En cuanto a operaciones geométricas en datos ráster se incluye: desplazamiento, escalado, rotación, deformación, agregación y desagregación. 
Finalmente, y no menos importante el libro aborda las operaciones entre ráster y vector, como: corte, extracción, rasterización y vectorización espacial; y la reproyección de datos geográficos.

La segunda parte se inicia con la elaboración de mapas, incluyendo estáticos, animados e interactivos. Adicionalmente, presenta una breve introducción al uso de shiny para aplicaciones de mapeo.

A continuación, en el capítulo 9, los autores abordan los métodos de enlace o puente a software GIS, como: QGIS, SAGA, GRASS; y, hacia bases de datos espaciales, como PostgreSQL/PostGIS.

El capítulo 10 presenta un resumen de la estructura de scripts, algoritmos geométricos y funciones.

El capítulo 11 aborda modelos computacionales y estadísticos con el objetivo de identificar patrones en los datos y realizar estimaciones a partir de ellos. Los autores presentan un estudio de caso relacionado con la estimación de la susceptibilidad a deslizamientos en el sur de Ecuador. Los autores hacen referencia al uso de la librería $m l r$, que incluye una cantidad importante de funciones en el ámbito del aprendizaje de máquina. En el estudio de caso, los autores usan, en primera instancia, la técnica de regresión denominada modelo lineal generalizado y la técnica de validación cruzada espacial con la finalidad de incorporar la característica de autocorrelación espacial que presentan los geodatos. Finalmente, explican el ajuste espacial de los hiperparámetros de la técnica de aprendizaje de máquina. Como punto a destacar, los autores hacen uso de funciones de paralelización para ejecutar los procesos de manera más rápida.

En la última parte del libro, los autores presentan algunos estudios de caso vinculados con los campos de la transportación, geomarketing y ecología.

Los autores mencionan que el libro pretende cubrir los aspectos críticos de la geocomputación, lo cual se cumple. Las técnicas de modelación e inferencia estadísticas abordadas se enfocan en aprendizaje de máquina y no cubren tópicos de estadística espacial y geoestadística, debido a que existen otros recursos especializados en esta temática.

El libro Geocomputation with $R$, constituye una excelente referencia de consulta para quienes están iniciando en la programación en el lenguaje y entorno de programación $\mathrm{R}$, así como para los profesionales que tienen conocimientos avanzados en la gestión de datos espaciales. Las funciones y temáticas abordadas en la publicación pueden ser aplicadas en pequeños proyectos, sin embargo, dada la potencialidad de la herramienta, se sugiere sugiero su aplicación en grandes volúmenes de datos, complementando su uso con las herramientas de procesamiento en paralelo y en la nube em la que actualmente se encuentran disponibles.

Otra gran potencialidad del uso de $\mathrm{R}$ en geocomputación es la posibilidad de incremento de nuevas funcionalidades debido al aporte colaborativo y constante de la comunidad de $\mathrm{R}$. 